\title{
A Decision Support System for railway timetabling (MOM): the Spanish case
}

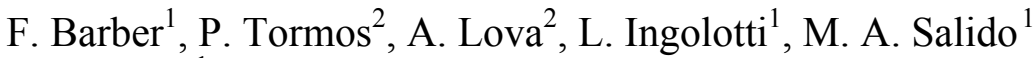 \\ \& M. Abril ${ }^{1}$ \\ ${ }^{1}$ DSIC, Universidad Politécnica de Valencia, Spain \\ ${ }^{2}$ DEIOAC, Universidad Politécnica de Valencia, Spain
}

\begin{abstract}
Train timetabling is a difficult and time-consuming task, given the complexity and number of constraints to be taken into account. A feasible train timetable should specify the departure and arrival time of each train to each location of its journey so that the line capacity and other operational constraints are met. Traditionally, train timetables have been generated manually by drawing trains on the time-distance diagram, where train schedules are manually adjusted so that all constraints are fulfilled. This process can take a long time and it usually stops once a feasible timetable is found. The difficulty of the process increases in complex and high-loaded networks and the resulting timetabling may be far from optimal. In this work, we present MOM: a friendly, flexible, computer-based Decision Support System (DSS) whose main goal is to support the railway planner to efficiently obtain optimized train timetables. The system generates feasible and high quality timetables by considering the set of constraints of the problem and optimization criteria related to the use of railway infrastructures and operator requirements. The railway planner can obtain several solutions automatically, by modifying certain parameters (departure interval, frequencies, among others). Hence, the user can analyze them and take decisions about conditions related to railway traffic and infrastructure. Furthermore, an important feature of the DSS is the consideration of hard and soft constraints which makes the search process more flexible and reaches better solutions. The developed DSS offers assistance in train scheduling, conclusions about the maximum capacity of the network, identifying bottlenecks, determining the consequences of changes, providing support in the resolution of incidents, and alternative planning in on-line scheduling for real traffic control. This DSS software is being successfully used by the Spanish Manager of Railway Infrastructure (ADIF).

Keywords: Decision Support System, train timetabling, railway capacity, on-line scheduling.
\end{abstract}




\section{Introduction}

European railways are undergoing a period of massive reforms that include the separation of infrastructure management from operators, which involves the open access to new operators competing for the use of the available infrastructure. This new scenario requires an improvement in the use of existing railway infrastructures, increased efficiency and the consideration of a more customer-oriented view looking for increasing the quality of the services they offer. The Spanish Manager of Railway Infrastructure (ADIF) is also involved in this process and is continuing the direction started some years ago, which is focused on the improvement of the use of the network capacity and the increase in quality of the services offered to customers. The Spanish railway network consists of $13,177 \mathrm{~km}$ of line that supports a heterogeneous traffic with around 5,000 daily trains, travelling a total of $494,230 \mathrm{~km}$ per day. The topology of the Spanish network is radial and is managed taking into account the scheduling of lines with few changeovers. Since is to make the best use of the available resources, one of the specific goals of ADIF is the automatic generation of train timetables that fulfil the existing constraints and optimize several quality criteria.

The MOM project is a long-term collaboration between the Polytechnic University of Valencia and ADIF. The aim of the project is the development of a Decision Support System that offers assistance in train scheduling, obtaining optimized timetables, conclusions about the maximum capacity of the network, identifying bottlenecks, determining the consequences of changes, providing support in the resolution of incidents, providing alternative planning, and real traffic control, etc.

There are several, useful software systems for railway planning management: RAILSYS, DONS, PETER, ROMAN, VIRIATO, CAPRES, FASTA, AFAIG, DEMIURGE, SISYFE, RAILCAP, OPENTRACK, OPENTIMETABLE, VISION, CMS, TPS and MULTIRAIL among others [2, 4]. All these systems have Infrastructure Management, Evaluation, and Simulation Management modules. Some of them have capabilities of on-line scheduling, timetable optimization, and capacity analysis. All these modules are integrated in MOM with different levels of development. Therefore, MOM can be considered as one of the most complete DSS software systems for railway timetabling, since it can support managers of railway infrastructure, in the difficult decision-making process they face.

\section{Railway problem description}

Given a railway line which may have both single and double-track sections, the railway timetabling problem consists in adding new trains (periodic or non periodic) to the line in such a way that the timetable assigned verifies the existing constraints and optimizes a multi-objective function. The railway line may be occupied by other trains whose timetables cannot be changed, and the new trains may belong to different operators with different priorities. The stations to be visited by each train may also be different from each other. The 
objective of the system is to obtain a feasible and optimized timetable taking into account: (i) the railway infrastructure topology, (ii) user requirements (parameters of trains to be scheduled), (iii) traffic safety rules, (iv) traffic previously scheduled on the same railway network, and (v) optimization criteria. Table 1 shows the main constraints that are taken into account by MOM.

Table 1: $\quad$ Constraints considered by MOM.

\begin{tabular}{ll}
\hline \multicolumn{1}{c}{ Railway Timetable Constraints } & \multicolumn{1}{c}{ Type } \\
\hline Time Interval for the Departure of the First Station & Operator \\
\hline Time Interval for the Arrival to the Final Station & Operator \\
\hline Maximum Delay allowed & Operator \\
\hline Frequency (for a set of periodic trains) & Operator \\
\hline Commercial Stops in Stations & Operator \\
\hline Journey Times (for each track section) & Operator \\
\hline Avoidance of Crossings (in one-way track sections) & Traffic \\
\hline Avoidance of Overtaking on track sections & Traffic \\
\hline Delay for a non specified commercial stop & Traffic \\
\hline $\begin{array}{l}\text { Reception/Expedition times in crossing/overtaking } \\
\text { operations. }\end{array}$ & Traffic \\
\hline Avoidance of Simultaneous Departures in stations & Traffic \\
\hline Finite track capacity of stations for traffic operations & Infrastructure \\
\hline Station Closing Times for traffic operation & Infrastructure \\
\hline Headway Times & Infrastructure \\
\hline
\end{tabular}

\section{General Architecture of MOM}

We have developed a flexible software system whose main goal is to assist the railway planner to design high-quality train timetables with a reasonable computational time. MOM generates feasible and high quality timetables by considering both the set of constraints of Table 1 and the multi-objective function specified. Moreover, the railway planner can also modify different parameters (departure interval, maximum delay, closing time, number of tracks, etc.) to adjust user/infrastructure requirements and obtain different solutions, analyze them, and take decisions about both railway traffic conditions and the available railway infrastructure.

Figure 1 shows the basic architecture of MOM. We have structured MOM in two main components: the interface and the optimizer. The interface allows the user to configure the scenario on which the optimization will be carried out; and the optimizer is responsible for providing the planner with a feasible, highquality timetable according to the constraints specified and the user requirements.

The interface module acquires the required railway infrastructure data directly from standard databases. The optimizer implements advanced Constraint Satisfaction and Optimization techniques to find feasible and high-quality timetables. A detailed description of the scheduling methods integrated in MOM 
is available in $[1,3,5]$. Once the first timetable is obtained, it is available to the user in a graphical or textual format, together with a detailed report about its main characteristics. Afterwards, MOM can obtain other successive and alternative timetables.

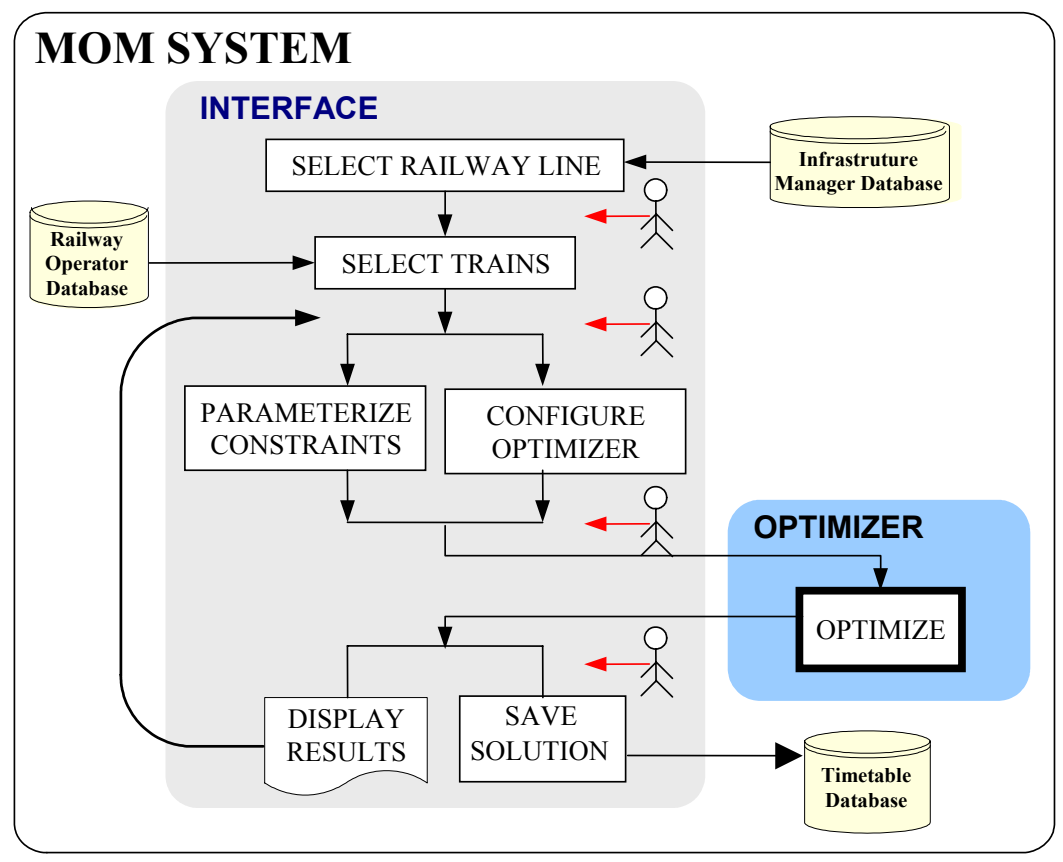

Figure 1: Components of MOM Interface.

In the interface module of MOM, the user can establish parameters corresponding to constraints, railway infrastructure, or trains. The main interface functions are described in the following points.

\subsection{Railway line and train selection}

The user selects the railway line to be used and the trains that will be taken into account by the optimizer. Some of these trains can be considered as trains in circulation. Therefore, their timetables are fixed and will not have to be modified during the solving process. The rest of the trains will make up the List of Trains to be Optimized (LTO), that is, the trains that need to be made compatible with the fixed trains over the given railway infrastructure.

\subsection{Journey, train and traffic constraints}

The main journey, train, and traffic constraints that the user can parameterize are shown in Table 1: the time interval for the departure of the first station and/or arrival to the final station for each train, the running time for each track section 
corresponding to the journey of each train in the LTO, maximum delay allowed for each train, the headway time between consecutive trains in the same direction, the reception and expedition times for crossing or overtaking operations, minimal time interval between consecutive departures in stations, the finite track capacity of stations, the closing time in stations for traffic operations, the frequency for periodic trains, the time intervals for infrastructure maintenance, the selection of locations for technical stops of some trains, and the delay for a non specified commercial stop due to unexpected acceleration and braking times. The main interface of MOM is shown in Figure 2.

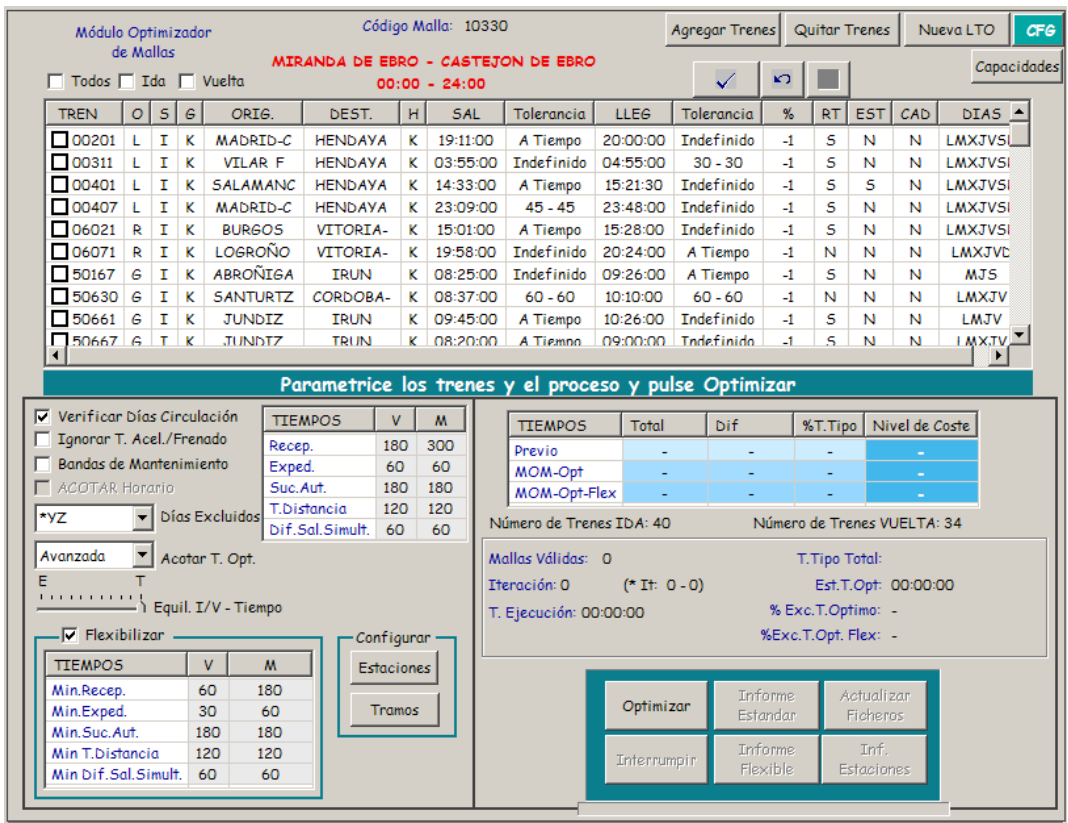

Figure 2: $\quad$ Main interface of MOM.

\subsection{Optimizer configuration}

The optimizer module uses a multi-objective function to assess the quality of feasible timetables. This function is composed by several weighted criteria: the average delay for each train, the number of traffic operations, the balance of the delay between upward and downward trains, etc. The weight assigned to each criterion is determined by user. According to the weight given to each criterion, the process searches for the solution that best fulfils the considered objective function. Furthermore, the user can bound the computational burden allowed for optimization. Hence, the obtained timetable will always be feasible, and it will be the best possible timetable according to the weighted optimization criteria and the allowed computational time. In this way, MOM can also be used as an "anytime scheduler". 


\section{Main capabilities of MOM}

MOM can optimize existing timetables, schedule and obtain automatically optimized timetables for new periodic and/or non-periodic trains and make them compatible with the existing scheduled trains. The system can also be used to reschedule timetables according to disruptions and delays in on-line, real-traffic management as well as validate and perform capacity analysis.

\subsection{Off-line railway planning}

MOM obtains optimized railway timetables in accordance with commercial/user requirements, railway infrastructure, traffic constraints, and optimization multicriteria. MOM is mainly configured as a computer-based support system for railway scheduling. Hence, the user can easily adjust several parameters (traffic constraints, infrastructure data, train data, journeys, etc.) and obtain correct and optimized timetables with different properties, allowing the user to make the final best selection. In addition, a given timetable could be interactively modified by the user, which can use MOM in order to validate its correctness.

\subsubsection{Flexible optimization}

MOM implements two optimization modes Standard Mode and Flexible Optimization Mode, which can be selected by the user in each optimization process. Standard Optimization (which is the default mode) implies the satisfaction of all the operator, traffic, and infrastructure constraints. In the Flexible Optimization mode, some constraints are considered as Soft Constraints, so that they can be relaxed if the resulting timetable is considerably improved. For instance, the travel time of a given train between two stations would be diminished by $3 \%$ in order to avoid a crossing operation with a train going in the opposite direction. Alternatively, the reception time could be relaxed 30 seconds in that station.

The constraints that can be relaxed in the flexible optimization mode are: the running time of a train in sections, and the temporal margins for reception, expedition, and headway. The user establishes the allowed relaxation bounds for these constraints.

The flexible optimization is applied by MOM if and only if (i) the user selects the Flexible Optimization mode, and (ii) the relaxation of the allowed soft constraints is justified with the improvement achieved in the timetable. After a flexible optimization is performed, a set of constraints is proposed to be relaxed. The user can select to validate all of the relaxed constraints, a subset, or none of them, according to viability of the proposed relaxations and the achieved improvement. All the relaxed constraints in a final timetable have to be validated by the user.

Figure 3a, illustrates a feasible timetable obtained by an expert planner, with a total travel time of 46:41:30. This timetable has been automatically optimized by applying the flexible optimization module of MOM. The total travel time is reduced by $5: 36: 05$, (total travel time: 41:05:00) by relaxing some constraints (Figure 3b). 


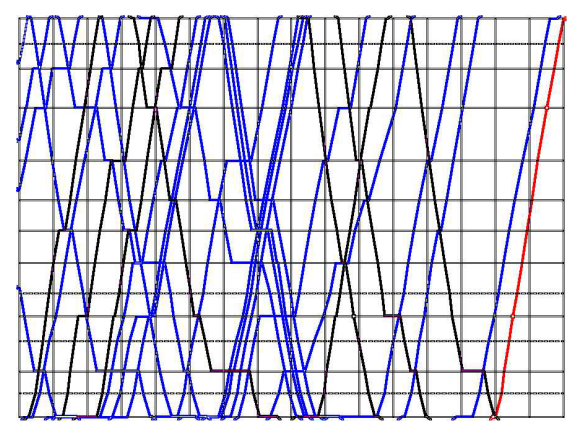

(a)

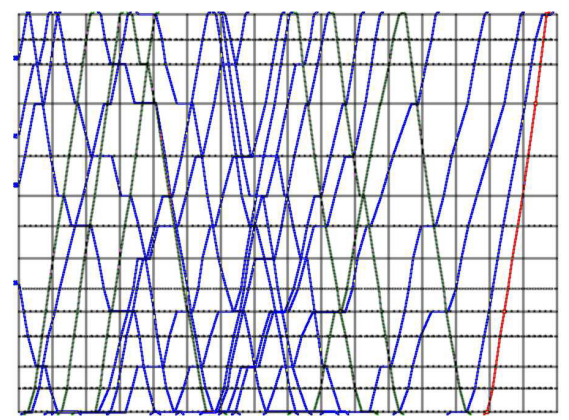

(b)

Figure 3: (a) A Standard-Optimized timetable. (b) Improved timetable using Flexible-Optimization.

\subsection{On-line railway planning}

MOM can be used to reschedule traffic timetables according to disruptions and delays in on-line and real-traffic management. The system supports the user in the decision making when disruptions occur. Two objectives can be selected in order to obtain an updated timetable after disruptions: (i) the minimization of the difference between the original timetable and the new timetable for each station and (ii) the minimization of the difference in scheduled arrival time of trains to the final station of their journeys. Figure 4 shows four incidences in a sample instance: two trains that are behind schedule and two trains that are ahead of schedule. After the application of the on-line railway planning module of MOM, the original timetable is updated taking into account the new situation.
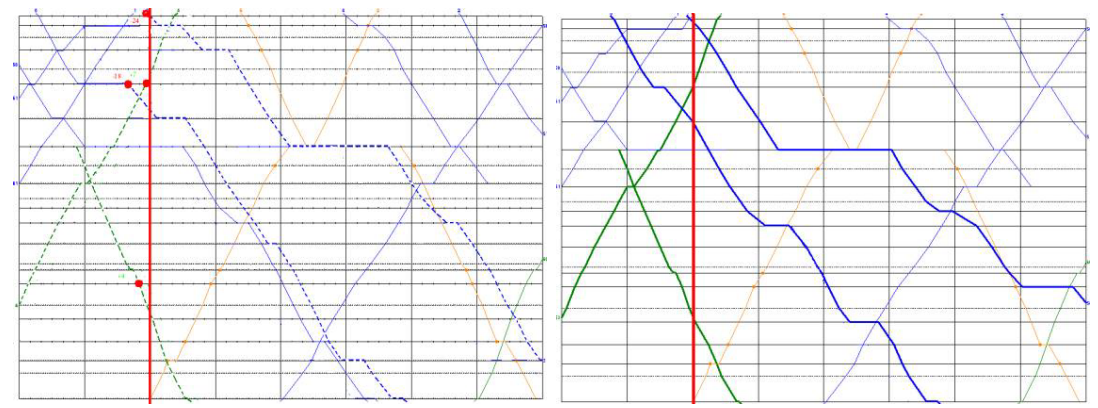

Figure 4: On-line scheduling.

\subsection{Railway capacity analysis}

Optimizing the use of railway infrastructure is a complex and difficult task. Questions such as "What part of extra traffic can be absorbed by the existing 
infrastructure?", "How much investment will be required for new infrastructure?" are important and difficult questions to answer.

The goal of capacity analysis is to determine the maximum number of trains that would be able to operate on a given railway infrastructure during a specific time interval, given the operational conditions. MOM can validate and perform capacity analyses of railway infrastructures. The systems can perform both theoretical and practical capacity analyses. In the first case, an analytical study is carried out, whereas the practical analysis is based on simulation (Figure 5). In both cases, if operational conditions and/or railway infrastructure change, the system is able to efficiently update the results of the analyses.

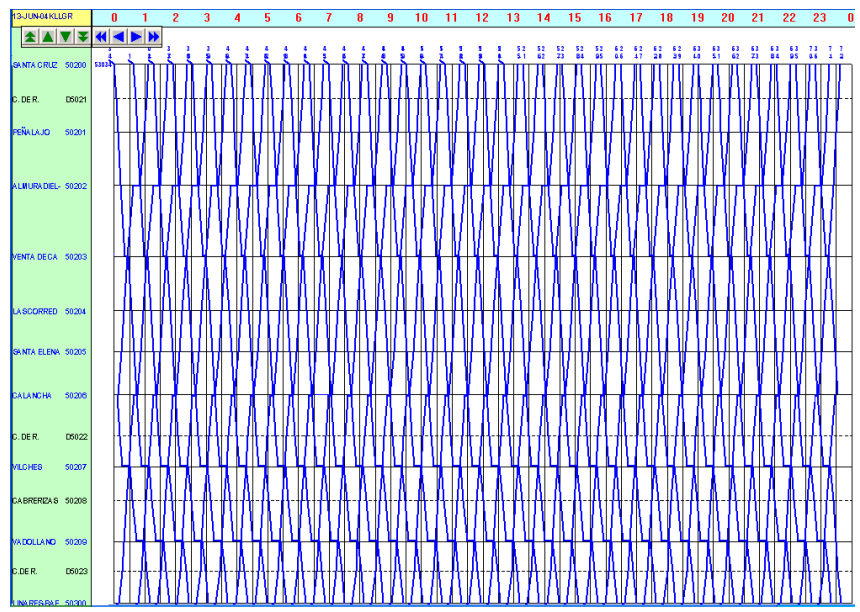

Figure 5: Analysis of line capacity.

\section{Some computational issues}

MOM is a very efficient software tool. The computational effort to obtain a satisfactory optimized solution depends on the number of trains, the railway infrastructure and its capacity (tracks in stations, single/double-way tracks), the required traffic operations due to the load of the network, etc.

Typically, the computational time required for very complex and real problems varies between a few seconds and 2-5 minutes. For instance, a railway timetabling problem that implies the scheduling of 95 new trains, with 37 trains already in circulation (with fixed timetables), on a line of $271.1 \mathrm{Kms}$, with 51 single-way track sections is optimized by MOM in less than 60 seconds. This scheduling problem implies the solution of 136 crossing conflicts. In this case, the search space is composed of $8.7 \mathrm{E}+40$ possible solutions. In order to reduce the search space, MOM makes use of several pre-processes, Operations Research techniques, and powerful, intelligent heuristics [3]. Moreover, several levels of optimization can be selected by the user. The minimum level obtains in a few seconds a feasible but middle-optimized timetable, so that the user can easily and 
efficiently evaluate different scheduling parameters and alternative timetables. The maximum optimization level obtains higher quality timetables when the computational time is longer. Figure 6 shows the optimization level with respect to the computational running time of three similar examples to the previous one.

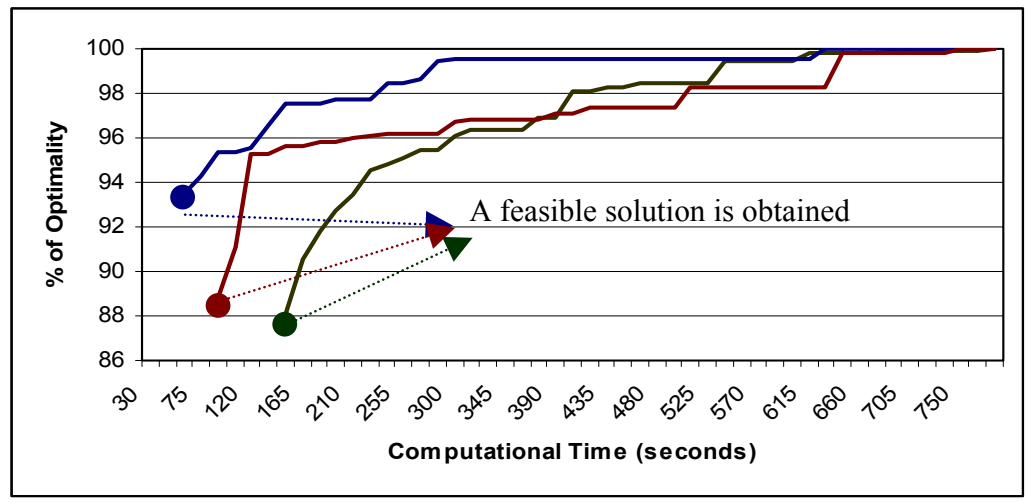

Figure 6: Optimization level vs computational time.

\section{Conclusions}

A Railway DSS is presented in this work. The system has three main scheduling modules:

i) Off-line Railway Planning, where the system solves and obtains optimized railway timetables in accordance with: commercial/user requirements, railway infrastructure, traffic constraints, and optimization criteria,

ii) On-line Railway Planning, which reschedules traffic timetables when disruptions, incidences, or delays occur, and

iii) Railway Capacity Analysis, which validates and performs capacity analyses of railway infrastructure.

MOM can be integrated in existing computer-based tools and obtains the required data from standard databases and friendly interfaces. This system is currently being used successfully by the Spanish Manager of Railway Infrastructure (ADIF) for obtaining off-line timetables. Currently, MOM is being improved adding the consideration of the analysis of the robustness and the stability of timetables generated. Further features of MOM can be obtained from http://www.dsic.upv.es/users/ia/gps/MOM.

\section{Acknowledgements}

This work has been partially supported by the research projects TIN2004-06354C02- 01 (Min. de Educación y Ciencia, Spain-FEDER), FOM-70022/T05 (Min. 
de Fomento, Spain) and FP6-021235-2, IST-STREP (UE). We appreciate the assistance of Jose Estrada (Spanish Administration of Railway Infrastructure ADIF).

\section{References}

[1] Barber, F., M. A.Salido; L. Ingolotti; M. Abril; A. Lova; P. Tormos., An InteractiveTrain Scheduling Tool for Solving and Plotting Running Maps. Current Topics in Artificial Intelligence. Lecture Notes in Computer Science/LNAI Series. Springer-Verlag. Vol. 3040, pp. 646-655, 2004.

[2] Hürlimann, D., "Object oriented modelling of infrastructure elements and business processes in railways". Ph D. Thesis. ETH Zurich.Switzerland, 2001.

[3] Ingolotti,L., F.Barber, P. Tormos, A. Lova , M. A. Salido, M. Abril, An Efficient Method to Schedule New Trains on a Heavily Loaded Railway Network. Advances in Artificial Intelligence. Lecture Notes in Computer Science/LNAI Series. Springer-Verlag Vol. 3315. pp. 164-173, 2004.

[4] Middelkoop, D. Bouwman, M. "Train network simulator for support of network wide planning of infrastructure and timetables. In: Computer in Railways VII, WIT Press, pp. 267-276. 2000.

[5] Salido, M.A., M. Abril , F. Barber, L. Ingolotti, P. Tormos, A. Lova., Topological Constraints in Periodic Train Scheduling. Frontiers in Artificial Intelligence and Applications. Planning, Scheduling and Constraint Satisfaction: From Theory to Practice. Frontiers in Artificial Intelligence and Applications. IOS Press. Vol. 117, pp. 11-20, 2005. 